\title{
Lattice Theory of Crystallization from a Melt and Volume Relaxation of Polymers
}

\author{
Takao IsHINABE and Kinzo IsHIKAWA \\ Faculty of Engineering, Tokyo Institute of Technology \\ Meguro-ku, Tokyo, Japan.
}

(Received July 17, 1971)

\begin{abstract}
A lattice theory is developed for crystallization of polymers from the melt containing seeded nuclei and for volume relaxation of amorphous (or molten) polymers. The partition function of a partially crystalline polymer is formulated on the basis of the lattice model; each end of the polymer chain is assumed to be included in the crystalline region and the middle part is in the amorphous region forming a folding loop or inter-lamellar chain. The Gibbs free energy is calculated from it. The equations describing the processes of crystallization and volume relaxation are derived by applying the linear law of irreversible thermodynamics to such systems of partially crystalline and molten polymers. The results of the theory are compared with available experimental data; a close agreement is obtained for crystallization with seeded nuclei, but the agreement is not satisfactory for volume relaxation except the very early stage of the process. The result can explain the depression of the melting temperature when the amorphous chains on the crystal surface deviate from the regular fold.
\end{abstract}

KEY WORDS Lattice Theory / Crystallization / Melt / Volume Relaxation / Partition Function / Free Energy / Irreversible Thermodynamics / Melting Temperature /

In a preceding paper $^{1}$, one of the authors developed a lattice theory for melting and glass transition of polymers; he derived the expressions of melting temperature and glass transition temperature in terms of molecular parameters, and discussed thermodynamic properties at the transition points. The present paper deals with crystallization kinetics with seeded nuclei and volume relaxation by applying irreversible thermodynamics and the lattice model.

Crystallization kinetics of polymers from the melt have been generally described in terms of the well-known Avrami equation ${ }^{2}$; it was originally for low-molecular-weight substances, but now is widely applicable to polymetric systems. Although many considerations ${ }^{3}$ have been given for the rate constant of this equation, the molecular ground for it is not yet sufficiently elucidated. In recent years, Roe and Krigbaum ${ }^{4}$ have applied the irreversible thermodynamics to their $\operatorname{model}^{5}$ for semi-crystalline polymers in order to obtain the relation corresponding to the Avrami equation.

As for the volume relaxation phenomena of polymers, phenomenological theories seem to be rather more prevalent than molecular ones. One of the latter theories has been developed by Hirai and Eyring ${ }^{6}$ on the basis of the hole model of liquids combined with the theory of rate process. On the other hand, Bueche ${ }^{7}$ has formulated the theory based on a simplified normal coordinate method for molecular motions in glass-forming substances; he led the equation for the rate of thermal contraction around the glass transition region.

We calculate the partition function of a partially crystalline polymer with the use of the lattice model, and obtain the Gibbs free energy of the system. Applying the linear law of irreversible thermodynamics to such systems of partially crystalline and molten polymers, we treat the crystallization with seeded nuclei and volume relaxation. The results of the theory are compared with available experimental data. 


\section{LATTICE MODEL}

Consider a system composed of $N$ symmetrical linear polymer chains with $n$ repeating units; the chain is split into $n^{\prime}$ segments, which move independently of each other. We pressume in both amorphous and crystalline states that the polymer chains are arranged in a hexagonal or tetragonal quasi-lattice with coordination number $z_{0}\left(z_{0}=4\right.$ or 6 according as tetragonal or hexagonal). In crystalline states they have the extended form and all the skeletal bonds are in trans conformation, whereas in molten or rubbery states holes are formed and the chain becomes flexible, that is, the bonds are in the state of mixture of trans and gauche conformations.

We now divide the system of volume $V$ into $N_{0}$ identical cells; $N n^{\prime}$ cells are occupied by segments and the other $n_{0}$ cells are vacant, i.e., $N_{0}=N n^{\prime}+n_{0} \quad\left(n_{0}=0\right.$ for the crystalline state). An effective sequence of gauche bonds constitutes a flexed segment, which gives a sharp bending of the chain; an unflexed segment is related to trans bonds. The relative fraction of flexed segments is assumed to have a value equal to that of gauche bonds. We consider the isothermal-isobaric ensemble of such a system. When $n^{\prime}$ is large enough, i.e., $n^{\prime} \simeq n^{\prime}-1$, the Gibbs free energy of a polymer in molten (or amorphous) state can be written in the form applying the lattice theory of a previous paper ${ }^{1}$ :

$$
\begin{aligned}
G_{\mathrm{a}}= & N n\left[\left(\frac{z_{0} u}{2}\right)(1-\phi)+\varepsilon_{\mathrm{T}}+\bar{f}\left(\varepsilon_{\mathrm{G}}-\varepsilon_{\mathrm{T}}\right)\right] \\
& -N n^{\prime} k T\left[\bar{f} \ln z_{0}\left(\frac{1}{\bar{f}}-1\right)-\ln \mathrm{e}(1-\bar{f})\right. \\
& \left.-\frac{\phi}{1-\phi} \ln \phi+\ln q_{\mathrm{a}}\right]+p V_{\mathrm{a}}
\end{aligned}
$$

$u$ being the inter-unit interaction energy, $\phi$ the volume fraction of holes $\left(\phi=n_{0} / N_{0}\right), \bar{f}$ the average relative fraction of gauche bonds, $\varepsilon_{\mathrm{T}}$ and $\varepsilon_{\mathrm{G}}$ the energies of trans and gauche conformations, and $q_{\mathrm{a}}$ the cell partition function for the amorphous state. The equilibrium value of $\phi$ is given by

$$
\ln \phi_{\infty}=-\left(1-\phi_{\infty}\right)\left[\left(-\frac{\nu^{\prime} z_{0} u}{2 k T}\right)\left(1-\phi_{\infty}\right)+1\right]-\frac{p v}{k T}
$$

where $\nu^{\prime}$ is the number of units per segment, i.e., $\nu^{\prime}=n / n^{\prime}$ and $v$ is the volume of a cell. Considering the "pentane interference" $\tilde{f}$ can be represented as ${ }^{1}$

$$
\bar{f}=\frac{f_{\mathrm{G}}}{1+\left(f_{\mathrm{G}}-f_{\mathrm{G}}{ }^{\prime}\right)}
$$

with

$$
f_{\mathrm{G}}=\frac{2 \exp [-\Delta \varepsilon / k T]}{1+2 \exp [-\Delta \varepsilon / k T]}
$$

and

$$
f_{\mathrm{G}}{ }^{\prime}=\frac{\exp [-\Delta \varepsilon / k T]}{1+\exp [-\Delta \varepsilon / k T]}
$$

where $\Delta \varepsilon$ is the energy difference between trans and gauche conformations; $\Delta \varepsilon=\varepsilon_{\mathrm{G}}-\varepsilon_{\mathrm{T}}$.

For a polymer in the crystalline state, the Gibbs free energy is written as, neglecting the chain folding :

$$
G_{\mathrm{c}}=N n\left[\left(\frac{z_{0} u^{\prime}}{2}\right)+\varepsilon_{\mathrm{T}}\right]-N n^{\prime} k T \ln q_{\mathrm{c}}+p V_{\mathrm{c}}
$$

Here $u^{\prime}$ is the inter-unit interaction energy in the crystalline state, and $q_{\mathrm{c}}$ the cell partition function of a crystalline polymer; one may put $u^{\prime}=u$ and $q_{\mathrm{c}}=q_{\mathrm{a}}$ around the melting point ${ }^{9}$.

\section{VOLUME RELAXATION}

Let an amorphous (or molten) polymer be initially in the equilibrium state at a given pressure and temperature. Either when a constant external pressure $p$ is applied to the polymer or when quenched to a constant temperature $T$, the volume contracts in some amount instantaneously and then decreases gradually to its new equilibrium value. The instantaneous contraction is considered to be due to the change in the volume of a cell in the lattice, while the time-dependent change may be the decrease of the number of holes in the system; the former is ascribed to thermal vibrations of the molecules around lattice points, and the latter to the micro-Brownian motion of the segments. We discuss here only the time-dependent volume change, namely volume relaxation*, except the

* Strictly speaking, we should use the term "volume retardation", but "volume relaxation" is more common. 


\section{T. IsHINABE and K. IsHIKAWA}

instantaneous one.

In general, the volume relaxation becomes remarkable near the glass-transition region since it requires a long time to reach the equilibrium state owing to the sluggish molecular motion. We choose the volume fraction $\phi$ of holes as the parameter characterizing the extent of the process; $1-\phi$ corresponds to the ordering parameter previously introduced by Davies and Jones $^{10}$ for glass-forming liquids. The affinity or the driving force for the volume relaxation process is defined by

$$
A_{1}=-\left(\frac{\partial G_{\mathrm{a}}}{\partial \phi}\right)_{T, p}
$$

Since $\phi \ll 1$ around $T_{\mathrm{g}}\left(\phi_{\infty}=0.025 \text { at } T_{\mathrm{g}}\right)^{11}$, the insertion of eq 1 into eq 7 gives

$$
A_{1}=-N n^{\prime} k T\left[\ln \phi+1+\frac{1}{k T}\left\{\left(-\frac{\nu^{\prime} z_{0} u}{2}\right)+p v\right\}\right]
$$

According to irreversible thermodynamics ${ }^{12}$, the rate of the irreversible process of a system is proportional to the affinity provided the system is not so far from equilibrium:

$$
\frac{\mathrm{d} \phi}{\mathrm{d} t}=L_{1} A_{1}
$$

with the proportionality constant $L_{1}$. By the use of the equilibrium value $\phi_{\infty}$ given by eq 2 , eq 9 is reduced to

$$
-\nu^{\prime} \frac{\mathrm{d} \phi}{\mathrm{d} t}=L_{1} \operatorname{Nnk} T \ln \left(\frac{\phi}{\phi_{\infty}}\right)
$$

The integration of eq 10 yields

$$
-\frac{t}{\tau}=\operatorname{li}\left(\frac{\phi}{\phi_{\infty}}\right)-\operatorname{li}\left(\frac{\phi_{0}}{\phi_{\infty}}\right)
$$

with

$$
\tau=\frac{\nu^{\prime} \phi_{\infty}}{L_{1} N n k T}
$$

Here $\phi_{0}$ is the volume fraction of holes at $t=0$ and li $x$ is the logarithmic integral function, defined by

$$
\text { li } x=\int \frac{\mathrm{d} x}{\ln x}=\ln |\ln x|+\sum_{r=1}^{\infty} \frac{(\ln x)^{\mathrm{r}}}{r \cdot r !}
$$

For the volume contraction of the case $\phi_{0} \gtrsim \phi \gtrsim \phi_{\infty}$, after expanding the right-hand side of eq 11 in the power series of $\left(\phi / \phi_{\infty}-1\right)$ and $\left(\phi_{0} / \phi_{\infty}-1\right)$, neglecting the higher order terms, we have

$$
-\frac{t}{\tau}=\ln \frac{\phi-\phi_{\infty}}{\phi_{0}-\phi_{\infty}}+\frac{\phi-\phi_{0}}{\phi_{\infty}}
$$

For an early stage of the process, i.e., $\left(\phi_{0}-\phi\right) / \phi_{\infty}$ $\ll 1$, excepting the instantaneous contraction, we approximately obtain

$$
V-V_{\infty}=\left(V_{0}-V_{\infty}\right) \exp \left(-\frac{t}{\tau}\right)
$$

where $V_{0}$ and $V_{\infty}$ are the volumes of a sample at $t=0$ and $t=\infty$, respectively. Equation 15 gives a linear relaxation process with single relaxation time $\tau$. This type of equation was derived by Hirai and Eyring ${ }^{6}$ on the basis of the theory of rate process for isothermal volume contraction of a polymer and liquid when the volume change is not so large.

Similarly, for the isothermal volume dilation due to decompression or heating of the conditions, $\phi_{0} \lesssim \phi \lesssim \phi_{\infty}$ and $\left(\phi-\phi_{0}\right) / \phi_{\infty} \ll 1$, we have

$$
V_{\infty}-V=\left(V_{\infty}-V_{0}\right) \exp \left(-\frac{t}{\tau}\right)
$$

\section{GIBBS FREE ENERGY OF A PARTIALLY CRYSTALLINE POLYMER}

Let us consider a partially crystalline polymer of crystallinity $\chi$ consisting of $N$ identically linked chains. The terminal parts of the polymer chain are included in crystal nuclei and fixed there; $\chi^{n}$ units are are in the crystalline region and the others $(1-\chi) n$ are in the amorphous region forming a folding loop or inter-lamellar chain. Each folding loop or interlamellar chain contains the same number of units and its distribution is not considered now. Hereafter we adopt the lattice model for the above system after Yamamoto and White ${ }^{13}$. The partition function may be given by putting $u=u^{\prime} \quad q_{\mathrm{c}}=q_{\mathrm{a}}(=q)$ :

$$
\begin{aligned}
Z= & q^{N n^{\prime}} \sum_{n_{0}} W\left(n_{0}\right) \exp \left(-\frac{E_{1}}{k T}\right) \\
& \times \exp \left(-\frac{E_{2}}{k T}\right) \exp \left(-\frac{p V}{k T}\right)
\end{aligned}
$$

with 


$$
\begin{aligned}
E_{1} & =N \nu^{\prime}\left(z_{0} n^{\prime}+2\right)\left[\chi+\left(1-\phi_{1-\chi}\right)(1-\chi)\right]\left(\frac{u}{2}\right) \\
E_{2} & =N \nu^{\prime}\left[\chi\left(n^{\prime}-1\right) \varepsilon_{\mathrm{T}}+(1-\chi)\left(n_{\mathrm{G}}{ }^{\prime} \varepsilon_{\mathrm{G}}+n_{\mathrm{T}}{ }^{\prime} \varepsilon_{\mathrm{T}}\right)\right] \\
& =N \nu^{\prime}\left(n^{\prime}-1\right)\left[\varepsilon_{\mathrm{T}}+(1-\chi) \bar{f} \Delta \varepsilon\right]
\end{aligned}
$$

and

$$
V=v\left(N n^{\prime}+n_{0}\right)
$$

Here $\phi_{1-\chi}$ is the volume fraction of holes in the amorphous region

$$
\phi_{1-\chi}=\frac{n_{0}}{(1-\chi) N n^{\prime}+n_{0}}
$$

and $W\left(n_{0}\right)$ is the number of ways of arranging $N$ molecules (each end being fixed) on the lattice of $(1-\chi) N n^{\prime}+n_{0}$ sites. The value of $W\left(n_{0}\right)$ is

$$
W\left(n_{0}\right)=\prod_{j=0}^{N-1} \frac{\left[(1-\chi)\left(n^{\prime}-1\right)\right] !}{2\left[(1-\chi) n_{\mathrm{T}}{ }^{\prime}\right] !\left[(1-\chi) n_{\mathrm{G}}{ }^{\prime}\right] !} \nu_{j+1}
$$

where $n_{\mathrm{G}}{ }^{\prime}$ and $n_{\mathrm{T}}{ }^{\prime}$ are the numbers of flexed and unflexed segments, and $\nu_{j+1}$ represents the number of ways for placing $(1-\chi) n^{\prime}$ segments, $(1-\chi) n_{G}{ }^{\prime}$ being flexed, of $j+1$-th chain on the lattice already containing $j$ chains. Following Flory $^{14}$ we have

$$
\begin{aligned}
\nu_{j+1}= & z_{0}{ }^{(1-\chi) n_{G^{\prime}}}\left[\frac{(1-\chi) n^{\prime}}{(1-\chi) N n^{\prime}+n_{0}}\right]^{(1-\chi) n^{\prime}} \\
& \times\left[\frac{(1-\chi) N n^{\prime}+n_{0}}{(1-\chi) n^{\prime}}-j\right]^{(1-\chi) n^{\prime}} \boldsymbol{P}_{j+1}
\end{aligned}
$$

where $\boldsymbol{P}_{j+1}$ is the probability that the $j+1$-th polymer chain has the end-to-end distance $r_{j+1}$. Introducing eq 23 into eq 22 and applying the Stirling approximation yield

$$
\begin{aligned}
W\left(n_{0}\right)= & \frac{1}{2^{\mathrm{N}}}\left[\frac{z_{0}{ }^{n_{\mathrm{G}^{\prime}}}}{e^{n^{\prime}}}\right]^{(1-\chi) N} \\
& \times\left[\frac{\left(n^{\prime}-1\right)^{n^{\prime}-1}}{\left(n_{\mathrm{T}}^{\prime}\right)^{n_{\mathrm{T}}}\left(n_{\mathrm{G}}\right)^{n_{\mathrm{G}^{\prime}}}}\right]^{(1-\chi) N} \\
& \times\left[\frac{(1-\chi) N n^{\prime}+n_{0}}{n_{0}}\right]^{n_{0}} \prod_{r} P_{\mathrm{r}}^{N_{\mathrm{r}}}
\end{aligned}
$$

where $N_{\mathrm{r}}$ is the number of amorphous chains with end-to-end distance $r$ having probability $P_{r}$. We presume Gaussian distribution ${ }^{15}$ for the amorphous chain:

$$
P_{\mathrm{r}}=v\left[\frac{3}{2 \pi(1-\chi) n^{\prime} b^{2}}\right]^{3 / 2} \exp \left[-\frac{3 r^{2}}{2(1-\chi) n^{\prime} b^{2}}\right]
$$

$b$ being the length of a segment.

We approximately evaluate the summation in eq 17 with the aid of the maximum term method. The generic term has the maximum when $\phi_{1-\chi}$ satisfies eq 2, i.e., $\phi_{1-\chi}=\phi_{\infty}$. With the wellknown relation

$$
G=-k T \ln Z
$$

we have the Gibbs free energy for a partially crystalline polymer:

$$
G=(1-\chi) G_{\mathrm{a}}+\chi G_{\mathrm{c}}-T \Delta S_{\mathrm{d}}
$$

Here $G_{\mathrm{a}}$ and $G_{\mathrm{c}}$ are given by eq 1 and eq 6 , and $\Delta S_{\mathrm{d}}$ is the entropy change caused by the deformation of the remaining amorphous chains induced by crystallization of the terminal parts. The value of $\Delta S_{\mathrm{d}}$ is given by

$$
\begin{aligned}
\Delta S_{\mathrm{d}}= & -\frac{3}{2} k N\left[\ln (1-\chi)+\frac{\left\langle r^{2}\right\rangle}{(1-\chi) n^{\prime} b^{2}}\right. \\
& \left.-\ln \frac{3 v^{2 / 3}}{2 \pi n^{\prime} b^{2}}\right]
\end{aligned}
$$

with

$$
\left\langle r^{2}\right\rangle=\frac{\sum_{r} r^{2} N_{\mathrm{r}}}{\sum_{r} N_{\mathrm{r}}}
$$

The mean square end-to-end distance $\left\langle r^{2}\right\rangle$ of the amorphous chain depends on the morphology of the respective crystalline polymers.

\section{CRYSTALLIZATION WITH SEEDED NUCLEI}

In this paragraph we deal with the crystallization caused by the subsequent deposition of the chain units in the amorphous regions on the

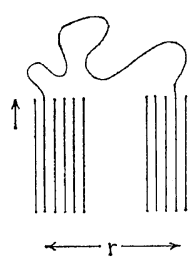

(a)

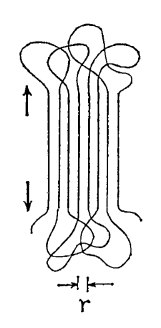

(b)

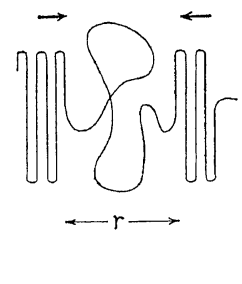

(c)
Figure 1. Models ${ }^{16}$ of crystal nuclei in a partially crystalline polymer; end-to-end distance $r$ of the amorphous chain is almost unchangeable while crystallization proceeds. The arrows indicate the growing directions of the nuclei. 
crystal nuclei already formed in the system. The nuclei are assumed to have such forms as (a) and (b), or (c) in Figure $1^{16}$; end-to-end distance $r$ of the amorphous chain is almost unchangeable during the process (in the case of (b), eq 24 holds with very rough approximation). We rewrite eq 27 in the following form neglecting the constant term:

$$
\Delta G^{\prime}=\chi \Delta G+\frac{3}{2} N k T\left[\ln (1-\chi)+\frac{\left\langle r^{2}\right\rangle}{(1-\chi) n^{\prime} b^{2}}\right]
$$

with

$$
\Delta G^{\prime}=G-G_{\mathrm{a}} \quad \text { and } \quad \Delta G=G_{\mathrm{c}}-G_{\mathrm{a}}
$$

In the case of the perfect crystal, we have at the melting point $\left(T_{\mathrm{m}}\right)$ :

$$
\Delta G=0 \text { or } \Delta H_{\mathrm{m}}=T_{\mathrm{m}} \Delta S_{\mathrm{m}}
$$

where $\Delta H_{\mathrm{m}}$ and $\Delta S_{\mathrm{m}}$ are the heat of fusion and the entropy of fusion. Therefore, eq $30 \mathrm{can}$ further be rewritten as

$$
\begin{aligned}
\Delta G^{\prime}= & -\chi \Delta H_{\mathrm{m}}\left(1-\frac{T}{T_{\mathrm{m}}}\right) \\
& +\frac{3}{2} N k T\left[\ln (1-\chi)+\frac{\left\langle r^{2}\right\rangle}{(1-\chi) n^{\prime} b^{2}}\right]
\end{aligned}
$$

The value of $\Delta H_{\mathrm{m}}$ is ${ }^{1,8}$

$$
\Delta H_{\mathrm{m}}=N n\left[\left(-\frac{z_{0} u}{2}\right) \phi+\bar{f} \Delta \varepsilon\right]
$$

In the temperatures below the melting point, crystallization proceeds, that is, the value of $\chi$ increases with time. If we choose $\chi$ as the parameter characterizing the extent of the process, the affinity of crystallization can be given by

$$
\begin{aligned}
A_{2}= & -\left(\frac{\partial \Delta G^{\prime}}{\partial \chi}\right)_{T, p} \\
= & \Delta H_{\mathrm{m}}\left(1-\frac{T}{T_{\mathrm{m}}}\right)+\frac{3}{2} N k T \\
& \times\left[\frac{1}{1-\chi}-\frac{\left\langle r^{2}\right\rangle}{(1-\chi)^{2} n^{\prime} b^{2}}\right]
\end{aligned}
$$

We presume the linear law of irreversible thermodynamics again:

$$
\frac{\mathrm{d} \chi}{\mathrm{d} t}=L_{2} A_{2}
$$

where $L_{2}$ is the proportionality constant for the process. The solution of eq 36 after substitution of eq 35 is

$$
\begin{aligned}
\beta t=\left(\chi-\chi_{0}\right) & +\gamma_{1} \ln \frac{\chi_{\infty}-\chi}{\chi_{\infty}-\chi_{0}} \\
& +\gamma_{2} \ln \frac{2+\delta-\chi_{\infty}-\chi}{2+\delta-\chi_{\infty}-\chi_{0}}
\end{aligned}
$$

with the abbreviations

$$
\begin{aligned}
& \beta=L_{2} \Delta H_{\mathrm{m}}\left(1-\frac{T}{T_{\mathrm{m}}}\right) \\
& \gamma_{1}=\frac{\delta}{2}\left[1-\frac{\delta+2\left\langle r^{2}\right\rangle / n^{\prime} b^{2}}{\delta+2\left(1-\chi_{\infty}\right)}\right] \\
& \gamma_{2}=\frac{\delta}{2}\left[1+\frac{\delta+2\left\langle r^{2}\right\rangle / n^{\prime} b^{2}}{\delta+2\left(1-\chi_{\infty}\right)}\right]
\end{aligned}
$$

and

$$
\delta=\frac{3}{2} R / n \Delta H_{\mathrm{m}}\left(\frac{1}{T}-\frac{1}{T_{\mathrm{m}}}\right)
$$

Here $\chi_{0}$ and $\chi_{\infty}$ are the crystallinities at $t=0$ and $t=\infty ; \chi_{\infty}$ is determined by the equilibrium condition $\left(\partial \Delta G^{\prime} / \partial \chi\right)_{T, p}=0$ :

$$
1-\chi_{\infty}=-\frac{\delta}{2}\left[\left(1+\frac{4\left\langle r^{2}\right\rangle}{\delta n^{\prime} b^{2}}\right)^{1 / 2}-1\right]
$$

In particular, for $\left\langle r^{2}\right\rangle \star n^{\prime} b^{2}$ and $\delta \ll 1$ these equations become approximately

$$
\beta t=\left(\chi-\chi_{0}\right)+\frac{1}{2}\left(1-\chi_{\infty}\right) \ln \frac{\left(\chi_{\infty}-\chi_{0}\right)\left(2-\chi_{\infty}-\chi\right)}{\left(\chi_{\infty}-\chi\right)\left(2-\chi_{\infty}-\chi_{0}\right)}
$$

and

$$
\chi_{\infty}=1-\left(\frac{\delta\left\langle r^{2}\right\rangle}{n^{\prime} b^{2}}\right)^{1 / 2}
$$

If we put $\left\langle r^{2}\right\rangle=n^{\prime} b^{2}$, they are equivalent to Roe and Krigbaum's theory ${ }^{4}$.

\section{COMPARISON WITH EXPERIMENT AND DISCUSSION}

\section{Volume Relaxation}

Kovacs ${ }^{17}$ carried out the experiments on isothermal volume contraction of several amorphous polymers using the temperature-jump method, and condensed the results into the following empirical relation over a medium time range:

$$
\frac{V-V_{\infty}}{V_{0}}=-b \ln \left(\frac{t}{t_{\mathrm{m}}}\right)
$$


where $b$ and $t_{\mathrm{m}}$ are the adjustable parameters. This equation represents neither exponential nor linear behavior. Recently, however, Goldbach and Rehage ${ }^{18}$ have measured the volume relaxation of polystyrene in detail by means of the pressure-jump method as well as the temperaturejump method. When the difference $V_{0}-V_{\infty}$ is not so large, the results show a linear relaxation, that is, $\left(V-V_{\infty}\right) /\left(V_{0}-V_{\infty}\right)$ does not depend on the value of $V_{0}-V_{\infty}$, and is the function of time only.

We will now compare our linear relation (eq 15) with their experimental data on volume relaxation of non-crosslinked polystyrene*; they were obtained by applying the pressure-jump method. Figure 2 illustrates the dependence of

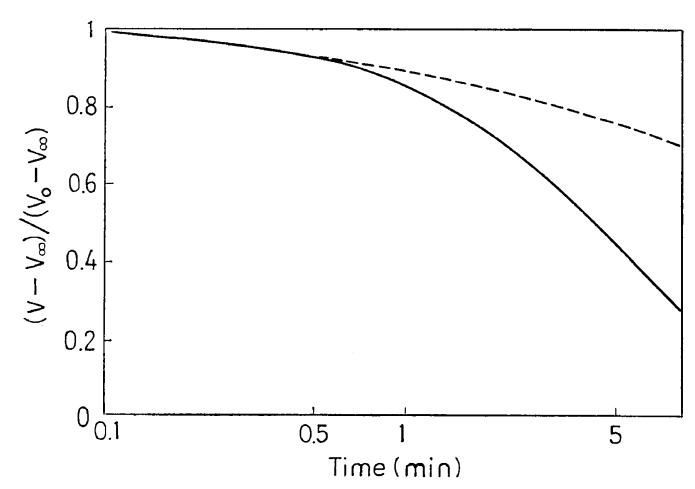

Figure 2. The dependence of $\left(V-V_{\infty}\right) /\left(V_{0}-V_{\infty}\right)$ on time. The full curve shows eq 15 with $\tau=6.12 \mathrm{~min}$. The dashed curve represents the experimental results of Goldbach and .Rehage ${ }^{18}$ for volume relaxation of non-crosslinked polystyrene.

$\left(V-V_{\infty}\right) /\left(V_{0}-V_{\infty}\right)$ on time. The dashed curve represents the experimental results at $T=91.84^{\circ} \mathrm{C}$ and $p=1 \mathrm{~atm}$, which were collected from the data obtained by different decompressions between 2.8 and $22.3 \mathrm{~atm}$. The full curve shows eq 15 with $\tau=6.12 \mathrm{~min}$; it coincides with the experimental curve in the very early stage of the process, but deviates conspicuously in the later stage. This discrepancy probably comes

\footnotetext{
* Strictly speaking, the present theory is not applicable to asymmetrical polymers such as polystyrene. However, we may apply the theory to such a polymer since volume fraction $\phi$ of the holes is independent of chain conformations of trans and gauche.
}

from the fact that such volume relaxation can not simply be described with a single relaxation time. It is necessary to introduce the distribution of relaxation times or the sizes of a segment [see eq 12]:

$$
V-V_{\infty}=\left(V_{0}-V_{\infty}\right) \int_{0}^{\infty} F(\tau) \exp \left(-\frac{t}{\tau}\right) \mathrm{d} \tau
$$

where $F(\tau)$ is the distribution function of relaxation time $\tau$.

\section{Crystallization}

We compare the present theory of crystallization with experimental data. The result is given in Figure 3; the open circles are the experimental values of Banks, et al. ${ }^{19}$ for a fractionated linear polyethylene sample which contains residual seeds $\left(\chi_{0}=0.0353\right)$ before it is crystallized at $127.5^{\circ} \mathrm{C}$. The full curve shows eq 43 when we put the parameters as $\chi_{\infty}=0.350$ and $\beta=0.02 \mathrm{~min}^{-14}$.

On the other hand, crystallization kinetics have usually been described by using the Avrami equation ${ }^{2}$ :

$$
\frac{\chi}{\chi_{\infty}}=1-\exp \left(-k t^{n}\right) .
$$

Here $k$ is the rate constant for crystallization, and $n$ is the integer between 1 and 4 (or half integer between them for diffusion-controlled growth),

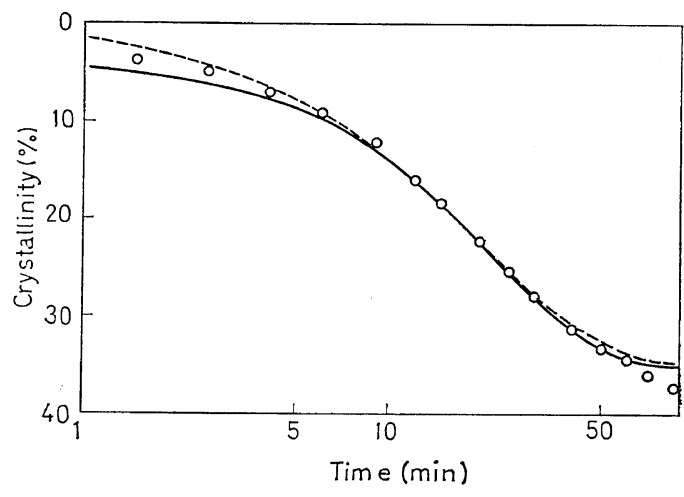

Figure 3. Change in crystallinity with time. The full curve shows eq 43 for $\chi_{\infty}=0.350$ and $\beta=0.02$ $\min ^{-1}$; the dashed curve the Avrami equation (eq 47) for $\chi_{\infty}=0.350, k=0.046 \mathrm{~min}^{-1}$, and $n=1$. The open circles are the experimental values of Banks, et al. ${ }^{19}$ for a fractionated linear polyethylene containing residual seeds. 


\section{T. IshinABE and K. IsHIKAwA}

whose value depends on the shape of the grawing nuclei. Applying the Avrami equation (eq 47) to the above case, we see theat the equation gives the best fit with the experimental values for the choice $n=1$; it corresponds to the heterogeneous nucleating system whose growth is linear. The dashed curve in Figure 3 shows eq 47 with such values of parameters as $\chi_{\infty}=0.35, k=0.046 \mathrm{~min}^{-1}$, and $n=1$; it is almost accordant with the curve of eq 43 except in the early part where a slight deviation is noticeable. These two curves give a close agreement with the experimental values in the crystallinity range below 35 per cent. In the range above this point, however, they considerably deviate from the experiment. It is probably because of the change in mechanism of the process due to the so-called secondary crystallization, as is frequently observed in the later stage of the process.

In the case $A_{2}>0$, crystallinity $\chi$ increases with time, whereas it decreases to zero for $A_{2}<0$, that is, the crystallites begin to melt at $A_{2}=0$. Hence, we can obtain the melting temperature $T_{\mathrm{m}}{ }^{\prime}$ of the reference sample from eq 42 by setting $\chi_{\infty}=0$ :

$$
\frac{1}{T_{\mathrm{m}}{ }^{\prime}}-\frac{1}{T_{\mathrm{m}}{ }^{0}}=\frac{3}{2} \frac{1}{n \Delta H_{\mathrm{m}}} \frac{\left\langle r^{2}\right\rangle}{n^{\prime} b^{2}}
$$

where $T_{m}{ }^{0}$ is the melting temperature of such crystal as $T_{\mathrm{m}}{ }^{0} / n^{\prime} b^{2}$ is almost equal to zero, i.e., the crystal with regular fold surface. This is very similar to the well-known relation given by Flory ${ }^{20}$, which represents the depression of the melting temperature when crystallite length does not attain its equilibrium value.

Since in eq $48, R T_{\mathrm{m}}{ }^{0} / n \Delta H_{\mathrm{m}} \ll 1$ for polyethylene, we can approximately obtain for $\left\langle r^{2}\right\rangle / n^{\prime} b^{2}$ $\$ 1$ after a simple calculation:

$$
T_{\mathrm{m}}{ }^{\prime}=T_{\mathrm{m}}{ }^{0}\left(1-\frac{3}{2} \frac{R T_{\mathrm{m}}{ }^{0}}{n \Delta H_{\mathrm{m}}} \frac{\left\langle r^{2}\right\rangle}{n^{\prime} b^{2}}\right)
$$

This equation shows that the melting temerature lowers as either the crystal deviates from regular folding or its thickness becomes small if we ignore the change in thickness of amorphous regions; the equation essentially accords with Lauritzen and Hoffman's equation ${ }^{21}$

$$
T_{\mathrm{m}}{ }^{\prime}=T_{\mathrm{m}}{ }^{0}\left(1-\frac{2 \sigma}{L \Delta H_{\mathrm{nl}}}\right)
$$

where $\sigma$ is the surface free energy of the crystal with lamellar thickness $L$. The values of $\sigma$ and $L$ are related to those of $\left\langle r^{2}\right\rangle / n^{\prime} b^{2}$ and $n$ in eq 49 , respectively.

Acknowledgment. The authors are much indebted to Professor M. Yamamoto of Tokyo Metropolitan University for many helpful discussions and a critical reading of the manuscript.

\section{REFERENCES}

1. T. Ishinabe, J. Phys. Soc. Japan, 30, 1029 (1971).

2. M. Avrami, J. Chem. Phys., 7, 1103 (1939); ibid., 8, 212 (1940); ibid., 9, 177 (1941). See also ref 3 .

3. L. Mandelkern, "Crystallization of Polymers", McGraw-Hill, New York, N.Y., 1964, Chap. 8.

4. R.-J. Roe and W. R. Krigbaum, Polymer, 6, 231 (1965).

5. R.-J. Roe, K. J. Smith, Jr., and W. R. Krigbaum, J. Chem. Phys., 35, 1306 (1961).

6. N. Hirai and H. Eyring, J. Appl. Phys., 29, 810 (1958), and J. Polym. Sci., 37, 51 (1959).

7. F. Bueche, J. Chem. Phys., 36, 2940 (1962).

8. J. L. White and M. Yamamoto, J. Phys. Soc. Japan, 28, 891 (1970).

9. T. Ishinabe, J. Phys. Soc. Japan, 30, 1022 (1971).

10. R. O. Davies and G. O. Jones, Proc. Roy. Soc. (London), A217, 26 (1953).

11. For example, F. Bueche, "Physical Properties of Polymers', Interscience, New York, N.Y., 1962, Chap. 5.

12. For example, S. R. De Groot, "Thermodynamics of Irreversible Processes", Interscience, New York, N.Y., 1951.

13. M. Yamamoto and J. L. White, J. Polym. Sci., Part A-2, 9, 1399 (1971).

14. P. J. Flory, J. Chem. Phys. 10, 51 (1942).

15. For example, P. J. Flory, "Principles of Polymer Chemistry", Cornell University Press, Ithaca, N.Y., 1953, Chap. 10.

16. H. G. Zachmann, Z. Naturforsch, 19a, 1397 (1964).

17. A. J. Kovacs, J. Polym. Sci., 30, 131 (1958).

18. G. Goldbach and G. Rehange, Rheol. Acta, 6, 30 (1967); J. Polym. Sci., Part C, 16, 2289 (1967).

19. W. Banks, M. Gordon, and A. Sharples, Polymer, 4, 289 (1963).

20. P. J. Flory, J. Chem. Phys., 17, 223 (1949).

21. J. I.Lauritzen, Jr. and J. D. Hoffman, J. Res. Nat. Bur. Stand., 64A, 73 (1960). 